\title{
Esophagitis dissecans superficialis: A case report and literature review
}

\author{
Shumona De MD, Geoffrey Williams MD FRCPC
}

$E^{s i n}$ ophagitis dissecans superficialis (EDS) is a rare condition characterized classically by sloughing of the esophageal mucosa, followed by vomiting or regurgitation of the esophageal cast (1). Since the advent of endoscopy, less dramatic cases of EDS have been diagnosed, characterized by stripped, whitish mucosa with or without bleeding, vertical fissures and circumferential cracks, in addition to long, linear mucosal breaks. EDS can be associated with medications such as bisphosphonates or nonsteroidal anti-inflammatory drugs, chemical irritants, celiac disease, hot beverages, autoimmune bullous dermatoses or idiopathy (2). EDS is known to be a benign condition that usually resolves without lasting pathological changes. Although biopsies from the esophageal mucosa of patients with EDS are sometimes associated with fungal or bacterial colonies (3), we report a case in which the appearance of EDS mimicked candidal esophagitis, and highlight the need to consider EDS in a differential diagnosis.

\section{CASE PRESENTATION}

An 81-year-old woman presented with a 20-year complaint of chronic intermittent dysphagia when swallowing solid food, accompanied by retrosternal chest pain lasting approximately $1 \mathrm{~min}$. Her medical history was significant for a cholecystectomy, appendectomy, partial hysterectomy and hemorrhoidectomy. She was being treated with pantoprazole $40 \mathrm{mg}$ daily. She had undergone upper gastrointestinal (UGI) endoscopy one year previously and endoscopic imaging was suggestive of candida esophagitis. Biopsies were unremarkable; however, the patient was treated for seven days with oral fluconazole but demonstrated minimal clinical improvement in her dysphagia.

Her symptoms did not fully resolve and she was referred to the department of gastroenterology for a repeat assessment. UGI endoscopy was repeated. A thin white membrane lifting from the mucosa was observed (Figure 1). Biopsies were taken and found to be consistent with mild candidal esophagitis. The patient was treated with fluconazole but experienced no improvement in symptoms.

UGI endoscopy was repeated two months later. The appearance of the esophagus had not changed. Biopsies taken during endoscopy (Figure 2) showed splitting of squamous mucosa at the suprabasal and superficial layers, with minimal focal lymphocytic infiltration at the deepest surfaces and diffuse parakeratosis throughout. No neutrophilic infiltrate was observed. In addition, no fungi were observed. This was believed to be consistent with a diagnosis of EDS.

The patient was treated by doubling the dose of pantoprazole to $80 \mathrm{mg}$ daily. The complaint of dysphagia improved on treatment. The patient was instructed to report any skin-related symptoms in case of an association with autoimmune disease. No other association with EDS was identified.

\section{DISCUSSION}

EDS is a rare but benign condition associated with certain medications, autoimmune conditions, irritants or idiopathy, and is most commonly diagnosed using endoscopy. EDS is characterized endoscopically by sloughing of the mucosal layer, which is apparent as sheets of mucosa (4). This can be confused with candidal esophagitis endoscopically; clinicians, therefore, rely on esophageal biopsies to confirm the

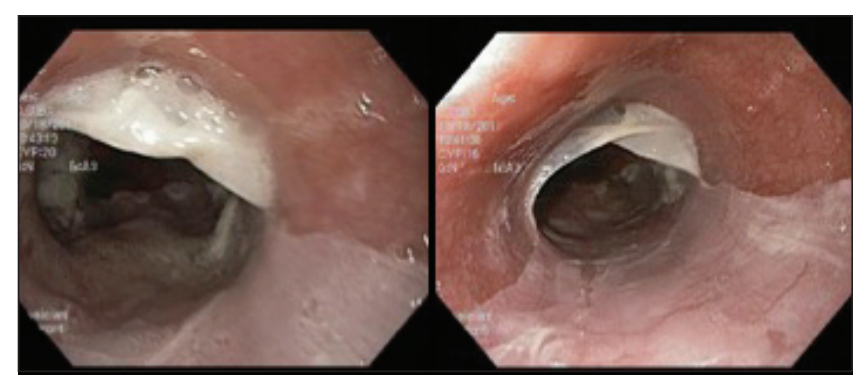

Figure 1) Endoscopy of the esophagus revealing sheets of mucosa sloughing off

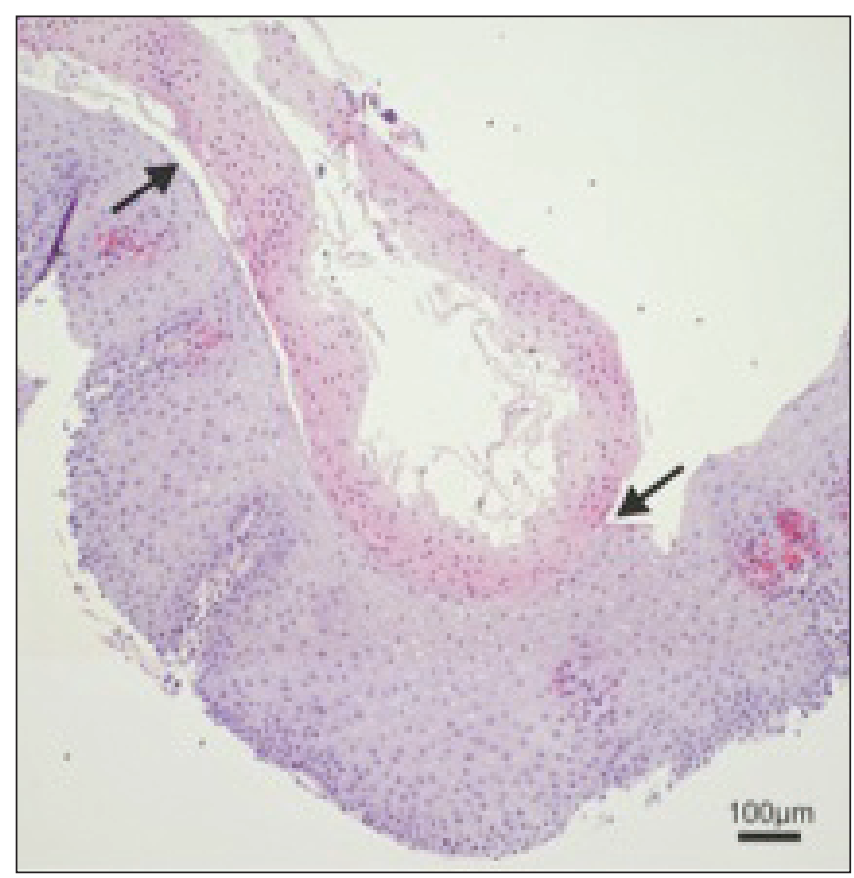

Figure 2) Biopsy of esophageal squamous mucosa showing a cleavage plane (arrows) near the top of the epithelial layer. Prominent parakeratosis in the desquamating layer is present. At the interface, minor degenerative change without inflammation is present. The basal layer is intact without degeneration or inflammation. Elsewhere in the biopsy there was minimal focal neutrophilic infiltrate at the postdesquamation epithelial surface and rare fungal hyphae and mixed bacterial flora colonies on the surface of the desquamated layer. Hematoxylin and eosin stain, original magnification $\times 100$

diagnosis. We emphasize the importance of considering EDS on a differential diagnosis during interpretation of both endoscopy and biopsy.

We report a case of EDS that was mistaken for candidal esophagitis. In this instance, the presence of Candida found on biopsy confirmed the false impression that the patient had candidal esophagitis, although it

Department of Medicine, Dalhousie University, Halifax, Nova Scotia

Correspondence: Dr Geoffrey Williams, Victoria General Hospital, Room 920, 1276 Southpark Street, Halifax, Nova Scotia B3H 2 Y9.

Telephone 902-473-4585, fax 902-473-5548, e-mail gswillia@dal.ca

Received for publication April 22, 2013. Accepted May 12, 2013 
was likely a commensal organism rather than a true infection given that there was no improvement of symptoms with anti-fungal treatment. The presence of Candida with no active inflammation in EDS has been reported elsewhere (3). EDS may be more common than it has been represented in the literature, but may be missed due to low awareness of this condition by endoscopists and pathologists.

Our case was unique in the response to proton pump inhibitor therapy. This has not been described in the existing literature of EDS; however, it is possible that EDS was an incidental finding and the patient's symptoms were secondary to gastroesophageal reflux disease. We believe this was unlikely given the lack of retrosternal burning in the patient's symptomatology and the lack of findings on esophageal biopsy relating to acid reflux. It is also possible that EDS is a dramatic and rare response to gastroesophageal reflux disease.

\section{REFERENCES}

1. Cesar WG, Barrios MM, Maruta CW, Aoki V, Santi GG. Oesophagitis dissecans superficialis: An acute, benign phenomenon associated with pemphigus vulgaris. Clin Exp Dermatol 2009;34:614.

2. Longman RS, Remotti H, Green PH. Esophagitis dissecans superficialis. Gastrointest Endosc 2011;74:403-4.

3. Carmack SW, Vemulapalli R, Spechler SJ, Genta RM. Esophagitis dissecans superficialis ("sloughing esophagitis"): A clinicopathologic study of 12 cases. Am J Surg Pathol 2009;33:1789-94.

4. Hokama A, Yamamoto Y, Taira K, et al. Esophagitis dissecans superficialis and autoimmune bullous dermatoses: A review. World J Gastrointest Endosc 2010;2:252-6. 


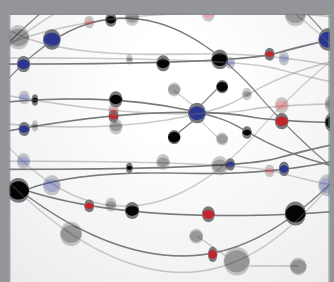

The Scientific World Journal
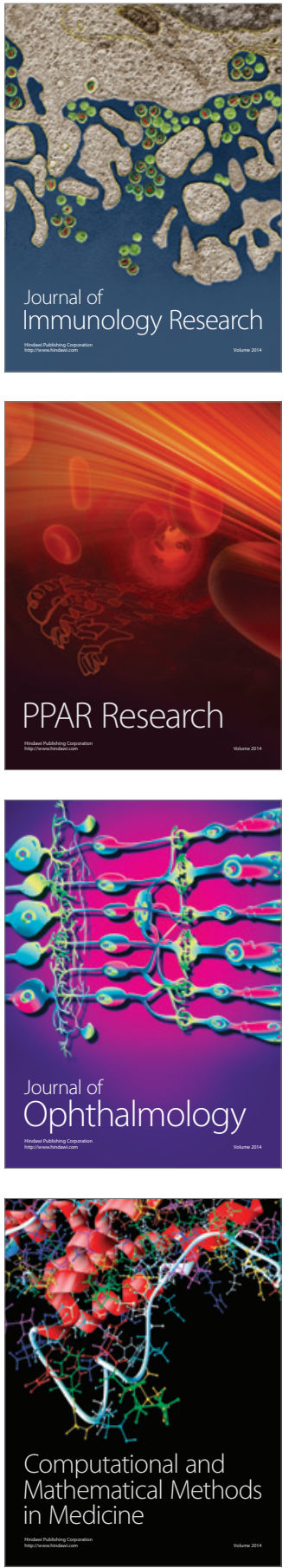

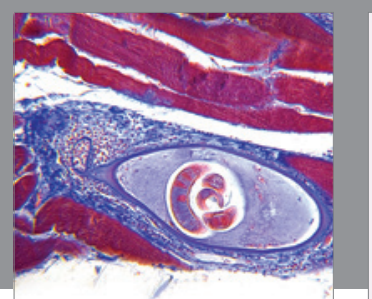

Gastroenterology Research and Practice

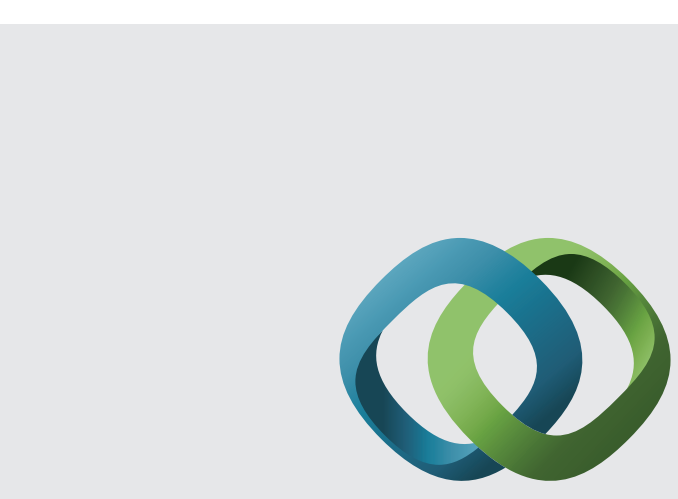

\section{Hindawi}

Submit your manuscripts at

http://www.hindawi.com
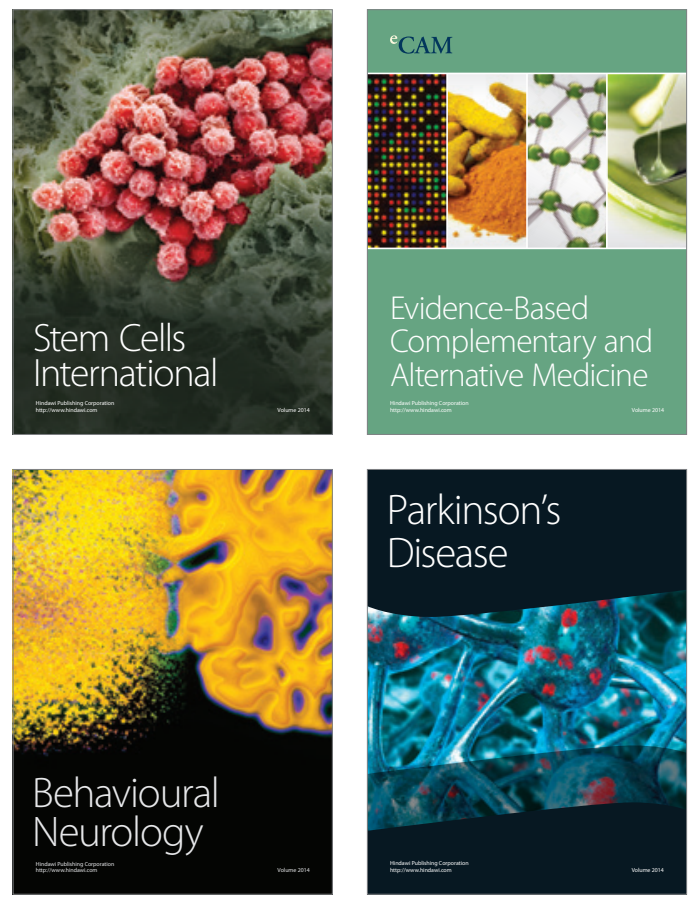
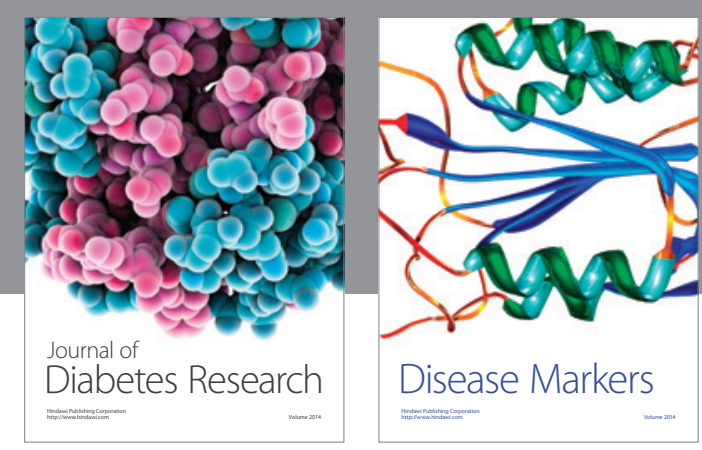

Disease Markers
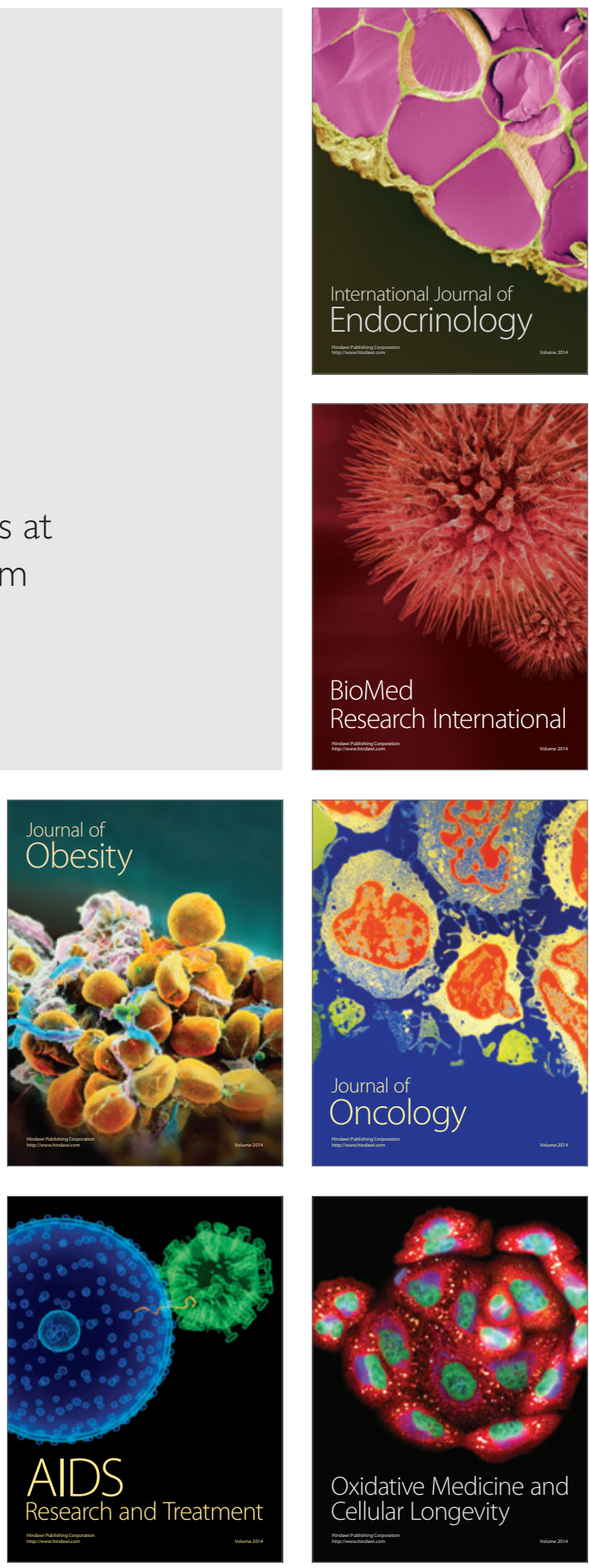